\title{
A comparative study on the electrical properties of different forms of carbon allotropes - epoxy nanocomposites
}

\author{
S. G. Stavropoulos, A. Sanida, G. C. Psarras*
}

Smart Materials \& Nanodielectrics Laboratory, Department of Materials Science, School of Natural Sciences, University of Patras, Patras 26504, Hellas (Greece)

Received 15 September 2019; accepted in revised form 9 January 2020

\begin{abstract}
In this study, series of nanocomposites consisting of an epoxy matrix and different carbon nanoinclusions (Carbon Black, Multiwall Carbon Nanotubes (MWCNT), Graphene nanoplatelets (GnP) and nanodiamonds) were developed, and their electrical response was examined in wide frequency and temperature ranges. Depending on the filler type and concentration, nanocomposites exhibited either insulator to conductor transitions or dielectric relaxation phenomena. Recorded relaxations were attributed to interfacial polarization, glass to rubber transition and motion of polar side groups. Nanocomposites integrating carbon black or MWCNTs exhibit an abrupt increase in permittivity and conductivity at a critical concentration (or percolation threshold). The insulator to conductor transition is described by means of percolation theory and critical concentration and exponent are determined. Conductance mechanisms are investigated in all sets of nanocomposites, by accounting the influence of temperature on conductivity and by applying the Variable Range Hopping model. Further, analysis reveals hopping conductivity as the main charge migration process below critical concentration, while hopping and metallic-like conduction coexist above it.
\end{abstract}

Keywords: nanocomposites, dielectric relaxations, conductivity, percolation, hopping

\section{Introduction}

Nanostructured carbon/polymer composites are in the focal point of the international research community for decades, because of their improved mechanical characteristics, enhanced heat resistance, and advanced optical and electrical behaviour. The unique properties of the allotropic modifications of carbon predetermine the wide variety of their application fields [1-10].

The majority of polymers are typically insulators with a very low concentration of free charge carriers and conductivity values ranging between $10^{-12}$ to $10^{-15} \mathrm{~S} / \mathrm{cm}$. Thus, they are considered as dielectrics and their electrical response is related to relaxation phenomena and can be described via the real and imaginary part of dielectric permittivity $[9,11]$. Integrating conductive inclusions, such carbon forms, in a polymer matrix, affect the resulting electrical properties and at a critical concentration, the composite's conductivity increases by several orders of magnitude.

Carbon lies in the IV column of the Periodic Table of Elements, differing from the other elements of the column by being able to form $\mathrm{sp}^{2}$ bonding. Carbon-reinforced polymer composites (CRPs) possess an outstanding position in the field of composites because of their applications in the areas of aerospace and automotive industries, sports and leisure goods, tires' industry, microelectronic, electrochemical manufacturing etc. The development, on an industrial scale, of high modulus and high strength carbon fibres, almost fifty years ago, was the initial impetus in CRPs applications, followed by the introduction of nanoscaled forms of carbon such as carbon nanotubes, graphene 
or carbon black. In all cases, the improved mechanical properties of CRPs was the leading property [2, $4,12-15]$. Although, nanoscaled forms of carbon is considered to exhibit mechanical properties of the order of TPa, only a limited portion of this ability is induced in the nano-CRPs. On the contrary, the effect of carbon nano-forms on the electrical response of CRPs appears to be more pronounced $[3,7,11$, $16,17]$. The term electrical response includes the dielectric properties and relaxation phenomena, the transition from the insulating to the conductive behaviour at a low critical concentration of carbon nanofiller, and the energy storage efficiency of the nanocomposites.

Besides a large number of publications upon the electrical response of various carbon forms/polymer nanocomposites and the existence of excellent reviews upon their performance $[3,7,12,18,19]$, there is a lack of comparative studies on the exclusive effect of different allotropes when all other parameters of the systems are kept constant.

In this work, four different nanoscaled carbon allotropes (carbon black, graphene nanoplatelets, multiwalled carbon nanotubes and nanodiamonds) were employed as reinforcing phase in an epoxy matrix, under identical contents and fabrication conditions, in order to investigate and compare the influence of each allotropic form of carbon upon the nanocomposites' electrical performance.

\section{Experimental}

In this study, four series of nanocomposites were developed varying the filler type and content. Sets of polymer nanocomposites were fabricated, by employing a two-component low viscosity commercially available epoxy resin (Epoxol 2004 provided by Neotex S.A., Athens, Greece) acting as the matrix. The type of the employed epoxy prepolymer was diglycidyl ether of bisphenol A (DGEBA) along with an aromatic amine as the curing agent. Four different forms of carbon allotropes were acting as filler namely

(a) carbon black nanoparticles with a mean diameter of $13 \mathrm{~nm}$ and a specific surface area approximately $550 \mathrm{~m}^{2} / \mathrm{g}$,

(b) graphene nanoplatelets with a thickness of 1-4 nm, particles' lateral size up to $2 \mu \mathrm{m}$ and specific surface area of $700-800 \mathrm{~m}^{2} / \mathrm{g}$,

(c) multi-walled carbon nanotubes with a length of $1-10 \mu \mathrm{m}(3-15$ walls), outer diameter 5-20 nm, inner diameter 2-6 $\mathrm{nm}$ and specific surface area $240 \mathrm{~m}^{2} / \mathrm{g}$ and

(d) nanodiamonds grade $\mathrm{G}$, with an average particle size of $4 \mathrm{~nm}$ and a specific surface area approximately $290-360 \mathrm{~m}^{2} / \mathrm{g}$.

The employed nanoinclusions were provided by Plasmachem $\mathrm{GmbH}$ and all the aforementioned physical properties are according to the manufacturer's datasheet.

The development of the nanocomposites included the addition of the nanoinclusions in the prepolymer under stirring in a sonicator for 10 minutes. Then the curing agent was added in a $2: 1 \mathrm{w} / \mathrm{w}$ mixing ratio and the stirring continued for another 10 minutes in the sonicator in order to avoid the formation of agglomerates, voids and bubbles. Subsequently, the mixture was poured into silicon moulds and cured at ambient for 7 days according to the provider's recommendations. After polymerization, the samples underwent a post-curing treatment at $100^{\circ} \mathrm{C}$ for 4 hours. Specimens were in the form of a circular disk with mean thickness $2.5 \mathrm{~mm}$ and diameter $35 \mathrm{~mm}$ and the filler content for all systems was: $0,0.1,1,3,5,7,10 \mathrm{phr}$ (parts per hundred resin per weight). The morphology of the produced specimens and the quality of the filler dispersion within the polymer matrix was assessed via Scanning Electron Microscopy employing an EVO MA 10 apparatus by ZEISS.

All four carbon nano-fillers were examined by means of Laser Raman Spectroscopy (LRS). LRS spectra were recorded by employing a micro-Raman system: HR800 (JY-Horiba Group). The excitation wavelength was $441.6 \mathrm{~nm}$, and the power of the laser beam was varying between 0.2 and $2 \mathrm{~mW}$, depending on the sample. The backscattered beam was selected using a $50 \times(\mathrm{NA}=0.55)$ microscope objective and directed to the entrance slit of the single monochromator after passing through an appropriate edge filter. Signals were recorded by an LN2-cooled 2DCCD detector. The spectral resolution was better than $3 \mathrm{~cm}^{-1}$.

The electric response of the nanocomposites was studied by means of Broadband Dielectric Spectroscopy using an Alpha-N Frequency Response Analyzer, under isothermal conditions, in the frequency range $10^{-1}-10^{7} \mathrm{~Hz}$ and temperature interval from 30 to $160^{\circ} \mathrm{C}$, with a $5^{\circ} \mathrm{C}$ temperature step controlled by Novotherm system. The heating rate between temperature steps was $0.5^{\circ} \mathrm{C} / \mathrm{min}$ and the stabilization time was $60 \mathrm{~s}$. The sample was placed inside 
the dielectric cell BDS 1200, mounted between two circular parallel gold-plated electrodes (with a diameter of 20 and $40 \mathrm{~mm}$ for the upper and lower electrode, respectively) forming a sandwich capacitor. The temperature of the sample was measured in realtime via the integrated Pt100 thermocouple right on the examined specimen with an accuracy of $\pm 0.1^{\circ} \mathrm{C}$ and the experimental data were recorded automatically via suitable software (Windeta). The dielectric cell was electrically shielded inside a suitable oven, in order to avoid measuring stray capacitances. The experimental procedure was in accordance with the ASTM D150 specifications for dielectric measurements. The amplitude of the time-varying voltage was equal to $1 \mathrm{~V}$ in all cases. Devices, as well as the dielectric cell and software, were supplied by Novocontrol Technologies.

\section{Results and discussion}

Figure 1 depicts representative SEM images for the specimens with $7 \mathrm{phr}$ carbon content. SEM images confirm the successful fabrication of the nanocomposites and their quality is deemed as satisfactory

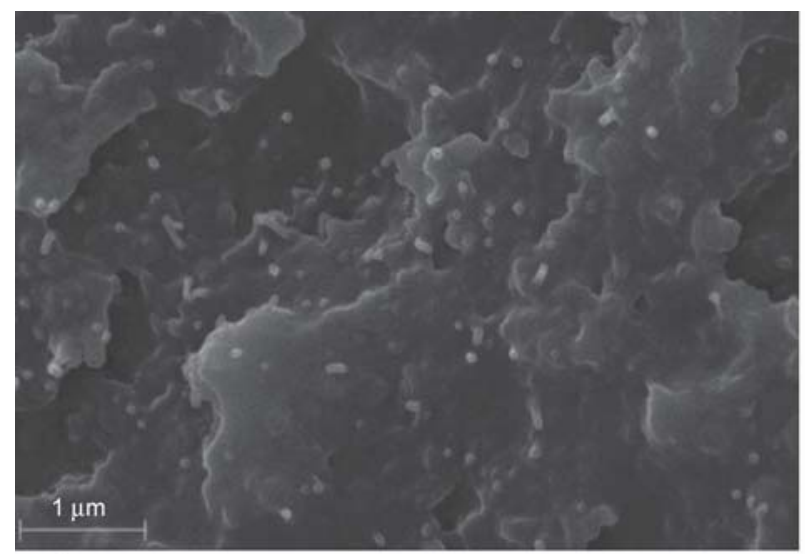

a)

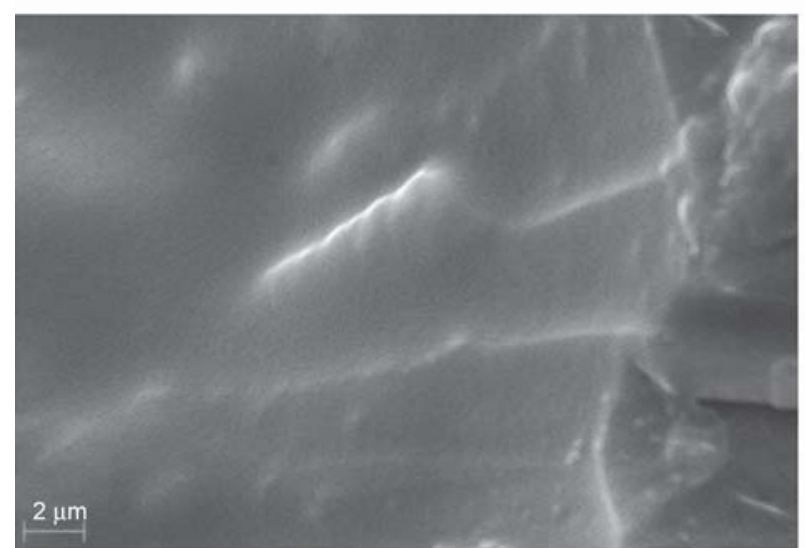

c) with fine nanodispersions of the carbonaceous fillers. Even at high concentrations, the formation of large agglomerates has generally been avoided. Interestingly, the MWCNTs are homogeneously dispersed within the polymer matrix and there are zones of high electron density with a reduced focus of the electron beam implying the formation of an interconnected network with potentially low contact resistance, Figure 1c.

LRS is considered as an effective experimental technique for investigating the 'fingerprint' of carbon allotropes [3] since recorded vibrations provide evidence for the present bonding. Obtained LRS spectra from all four carbon nano-filler, not shown here because of the journal's space limitations, include peaks at approximately 1350,1580 and $2700 \mathrm{~cm}^{-1}$ referred as D, G and 2D bands, respectively. $\mathrm{D}$ band is related to $\mathrm{sp}^{3}$ carbon atoms and its presence is indicative of disorder in the atomic arrangement or edge effects of graphitic layers. $G$ band originates from the in-plane vibration of the $\mathrm{sp}^{2}$ carbon atoms, while 2D band arises from second-order Raman scattering $[3,20]$.

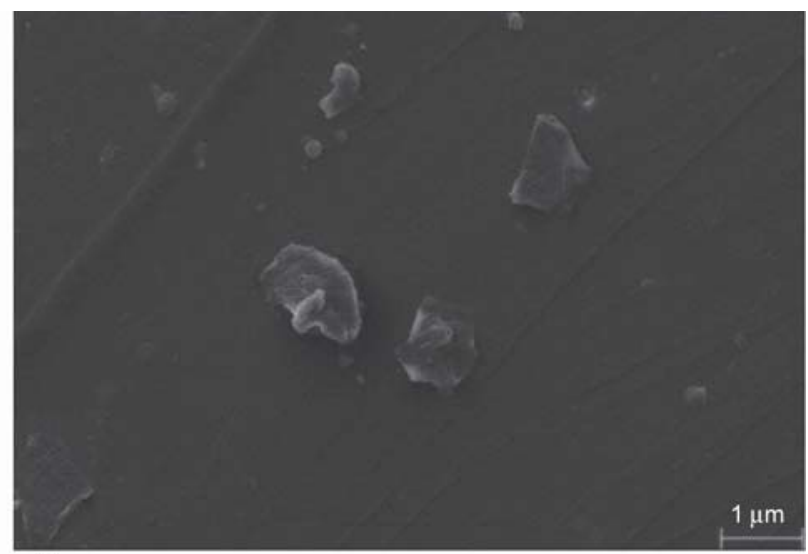

b)

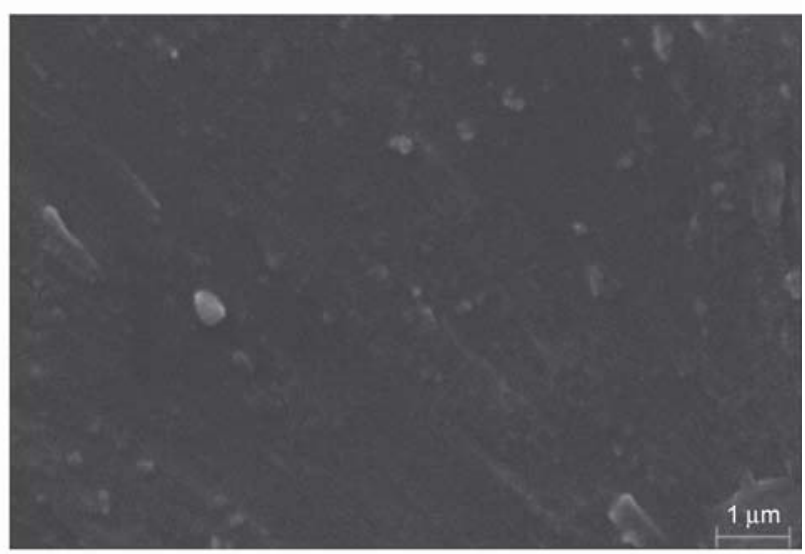

d)

Figure 1. SEM images for the nanocomposites with $7 \mathrm{phr}$ (a) carbon black, (b) GnP, (c) MWCNT and (d) nanodiamonds filler content. 
The variation of the real part of dielectric permittivity as a function of temperature and frequency for all nanocomposites with $3 \mathrm{phr}$ filler content is depicted in Figure 2. In all cases, $\varepsilon^{\prime}$ increases with temperature because thermal agitation enhances the chain flexibility and thus facilitates the alignment of the dipoles with the externally applied electric field. The real part of dielectric permittivity diminishes rapidly with increasing frequency since permanent and induced dipoles don't possess the necessary time to follow the field's alternation. A step-like transition recorded at intermediate frequencies and temperatures is ascribed to the $\alpha$-relaxation process, which is related to the glass to rubber transition of the polymer matrix. This behaviour is representative of all examined systems.

In the representative plots of the loss tangent versus frequency and temperature for the same nanocomposites (Figure 3), three distinct relaxation mechanisms were identified for all systems, that are associated
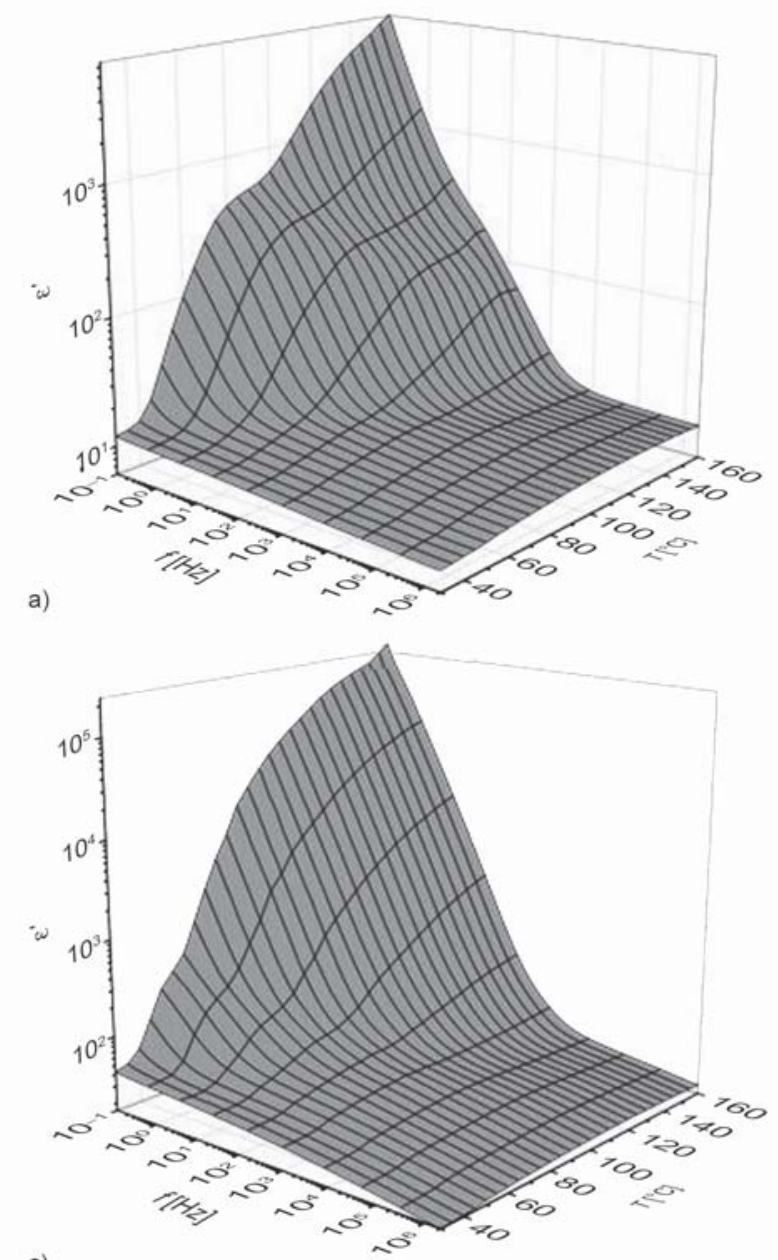

with both filler and the polymer matrix. The faster and also weaker mechanism recorded at high frequencies is attributed to the rearrangement of the small polar side groups of the polymer chain ( $\beta$-relaxation). At intermediate frequencies and temperatures, the $\alpha$-relaxation process is identified and is attributed to the transition of the polymer matrix from the glassy to the rubbery state. Finally, the slowest relaxation process recorded at low frequencies and high temperatures is attributed to interfacial polarization (IP), also known as Maxwell-Wagner-Sillars effect (MWS), where free charges accumulate at the interface between the filler and the matrix-forming large electric dipoles which attempt to follow the orientation of the applied field.

Comparative plots of the real part of the dielectric permittivity versus frequency at $30^{\circ} \mathrm{C}$, at various filler loadings for all examined systems, are depicted in Figure 4. Values of $\varepsilon^{\prime}$ systematically increase with filler content in the whole frequency range, mainly,
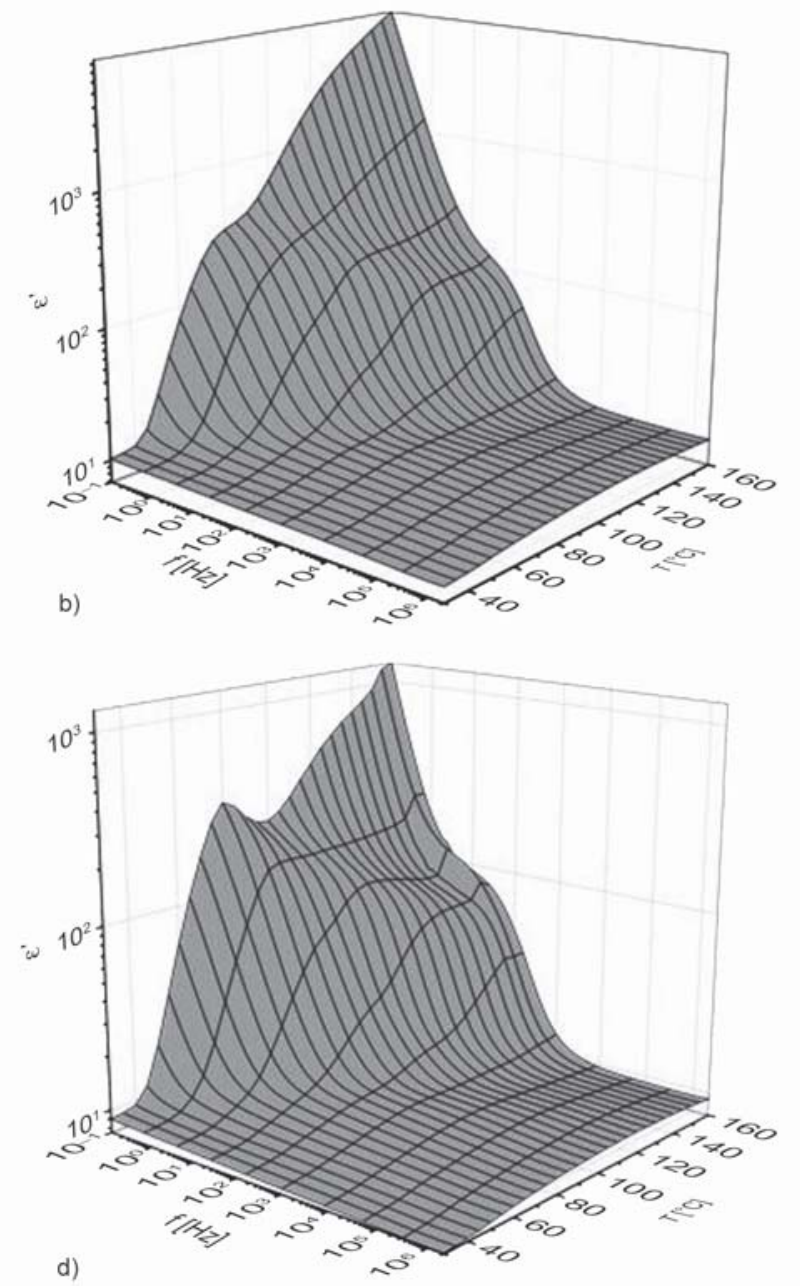

Figure 2. The variation of the real part of dielectric permittivity as a function of frequency and temperature for the nanocomposites with $3 \mathrm{phr}$ (a) carbon black, (b) GnP, (c) MWCNT and (d) nanodiamonds filler content. 

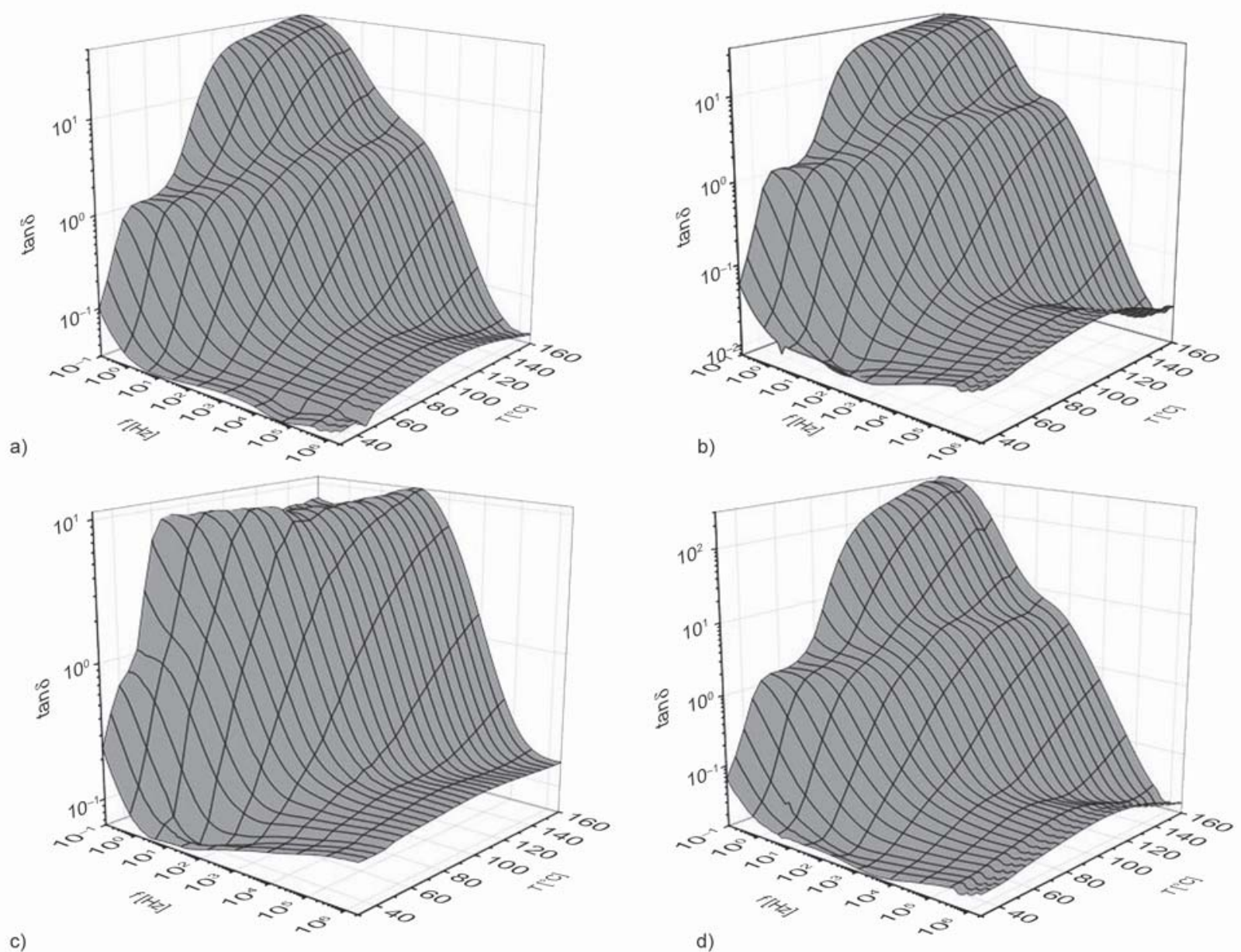

Figure 3. The variation of the loss tangent as a function of frequency and temperature for the nanocomposites with 3 phr (a) carbon black, (b) GnP, (c) MWCNT and (d) nanodiamonds filler content.

due to the enhanced conductivity of the carbon nanofillers for all systems, with the exception of nanocomposites filled with nanodiamonds. For this particular system, $\varepsilon^{\prime}$ attains its highest values at low filler content and then remains virtually the same and/or even diminishes with further filler loading, possibly as a result of the relative low conductivity of nanodiamonds (in diamond appear the $\mathrm{sp}^{3}$ bonding of tetrahedral structure, in contrary to the $\mathrm{sp}^{2}$ bonding of the planar structure of graphite) and their tendency to form agglomerates. The extremely high values of $\varepsilon^{\prime}$ at high filler content for the carbon black and MWCNT filled systems, attributed to the enhanced electrical heterogeneity between the insulating matrix and the highly conductive nanoinclusions, provide indirect indications of an insulator to conductor transition. The deviation from the pure insulating behaviour becomes evident by the absence of data for permittivity at low frequencies for the CNT composites and in the inset diagrams of Figure 4, which depict the real part of electric modulus as a function of frequency at $160^{\circ} \mathrm{C}$ at various filler loadings for all examined systems. In order to eliminate the parasitic phenomenon of electrode polarization dielectric data are presented into the electric modulus formalism [21-25]. Electric modulus is defined as the inverse quantity of the complex dielectric permittivity [21], as shown by Equation (1):

$$
M^{*}=\frac{1}{\varepsilon^{*}}=\frac{\varepsilon^{\prime}}{\varepsilon^{\prime 2}+\varepsilon^{\prime 2}}+i \frac{\varepsilon^{\prime \prime}}{\varepsilon^{\prime 2}+\varepsilon^{\prime 2}}=M^{\prime}+i M^{\prime \prime}
$$

where $\varepsilon^{\prime}, M^{\prime}$, and $\varepsilon^{\prime \prime}, M^{\prime \prime}$ are the real and imaginary parts of dielectric permittivity and electric modulus, respectively. Electric modulus formalism has been proved suitable for describing dielectric relaxation processes, especially in the low-frequency range and at high temperatures, by suppressing the enhanced values of $\varepsilon^{\prime}$ and $\varepsilon^{\prime \prime}$ thus neglecting the influence of electrode polarization upon dielectric data, [21-25]. Conductive systems exhibit extremely low values of $M^{\prime}$. The step-like transitions at intermediate frequencies, recorded in the $M^{\prime}$ spectra are related to the $\alpha$-relaxation mechanism. 

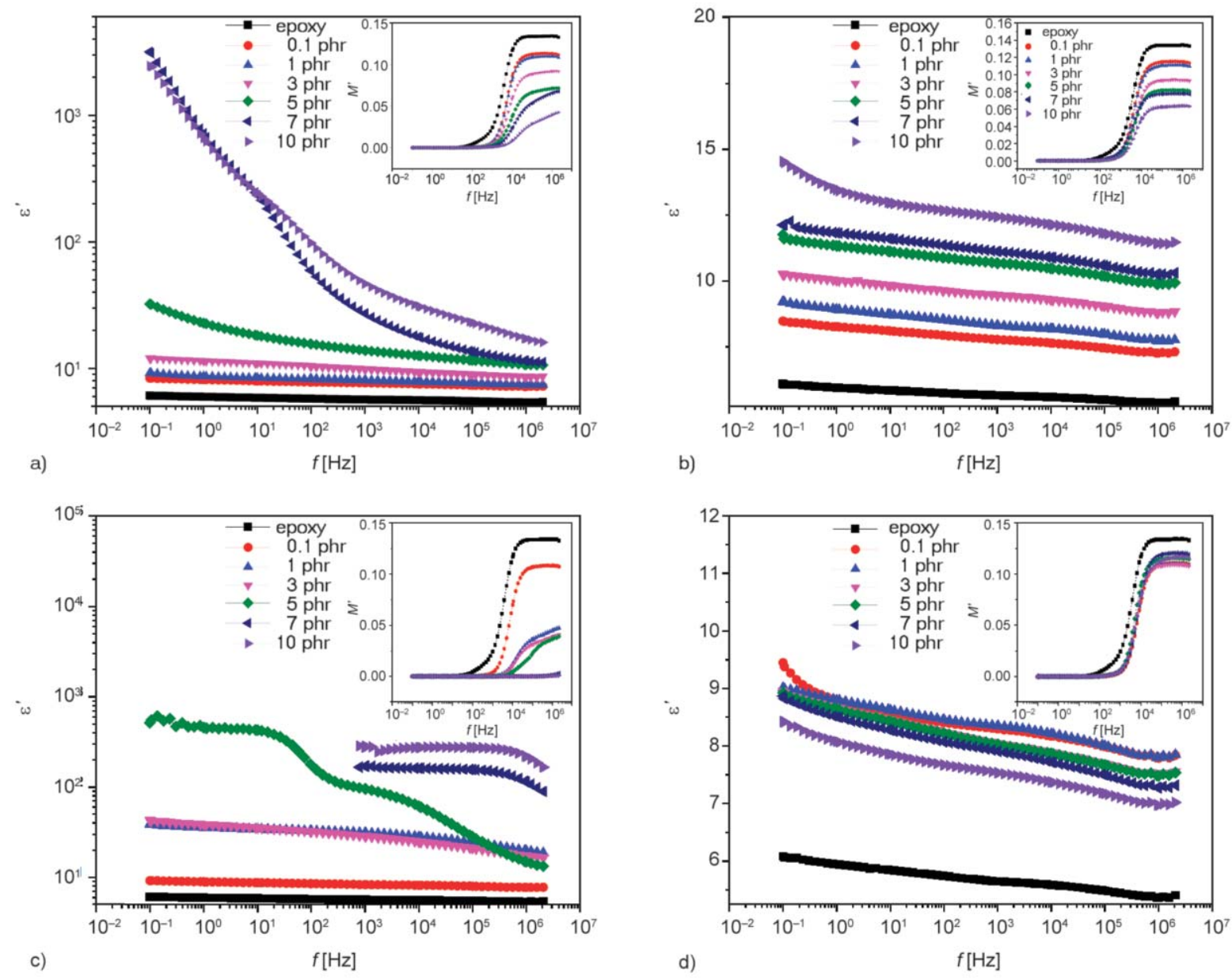

Figure 4. Real part of dielectric permittivity as a function of frequency, at $30^{\circ} \mathrm{C}$, for the nanocomposites with: (a) carbon black, (b) GnP, (c) MWCNT and (d) nanodiamonds nanoparticles. Insets depict the real part of the electric modulus as a function of frequency, at $160^{\circ} \mathrm{C}$ for the respective nanocomposites.

Electrical conductivity is the property of polymer nanocomposites most affected by the inclusion of carbon nanoparticles. AC conductivity has been calculated from the recorded dielectric loss according to Equation (2):

$\sigma_{\mathrm{AC}}(\omega)=\sigma=\operatorname{Re}\left[i \varepsilon_{0} \omega \varepsilon^{*}(\omega)\right]=\varepsilon_{0} \omega \varepsilon^{\prime \prime}$

where $\varepsilon_{0}$ is the permittivity of free space, $\varepsilon^{\prime \prime}$ the dielectric losses and $\omega$ the angular frequency of the field.

Figure 5 depicts comparative plots of the ac conductivity versus frequency at $30^{\circ} \mathrm{C}$ for all examined systems. Polymer nanocomposites are disordered solid materials, and their conductivity follows Equation (3), which is known as the ac universality law:

$\sigma_{\mathrm{AC}}(\omega)=\sigma_{\mathrm{DC}}+A(\omega)^{\mathrm{s}}$

where $\sigma_{\mathrm{DC}}$ is the dc conductivity $(\omega=0)$ and $A, s$ are parameters depending on temperature and filler content. Above a critical frequency, AC conductivity increases with frequency, because localized charge carriers hop between adjacent conductive sites, instead of attempting to migrate to larger distances within the nanocomposite, while being confined by the insulating matrix, at low frequencies.

$\mathrm{AC}$ conductivity increases with filler content due to the higher conductivity values of the nanocarbon inclusions. Moreover, the critical frequency seems to shift to higher frequencies with increasing filler content. The abrupt increase of several orders of magnitude for the carbon black and the MWCNT systems along with the frequency-independent behaviour for the high filler content of these systems signifies a clear indication for the transition from the insulating to the conductive behaviour of the respective nanocomposites.

This significant increase of conductivity corresponds to a very narrow variation of the conductive phase content and can be described by means of percolation theory, which predicts that at a critical concentration (also called percolation threshold) a conductive path 

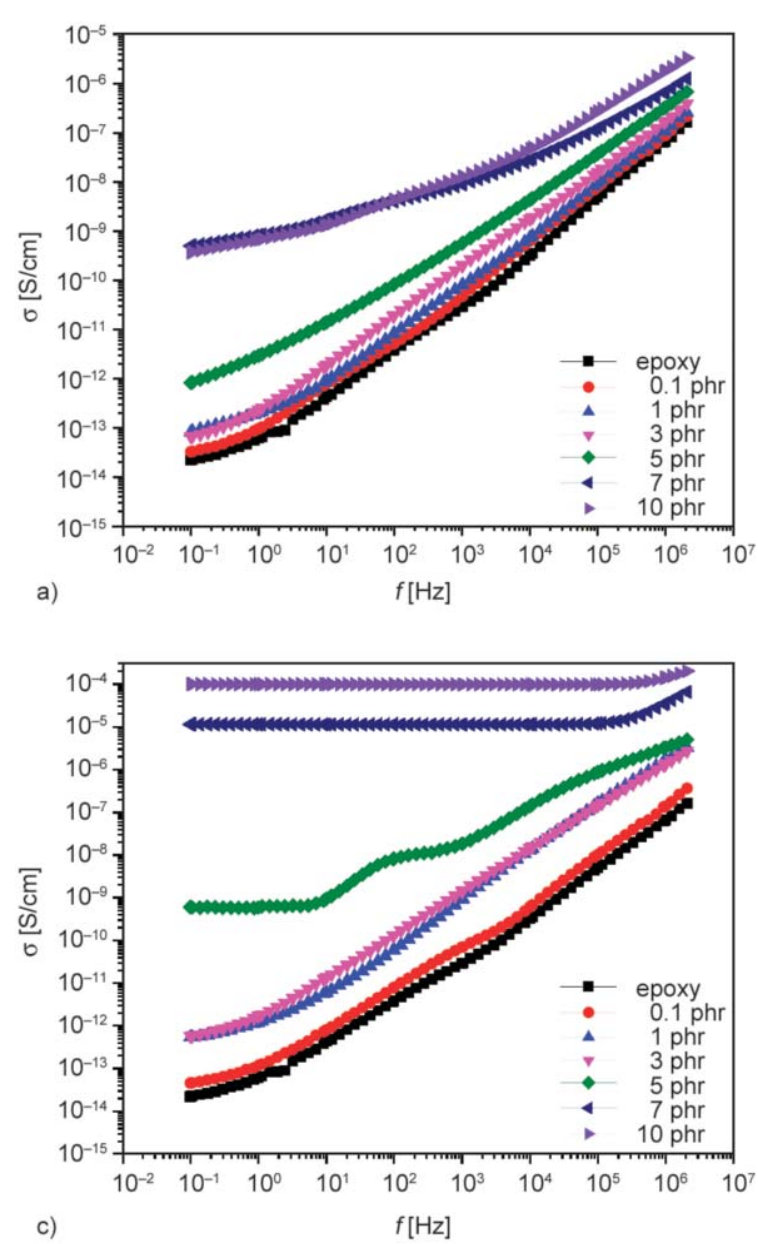
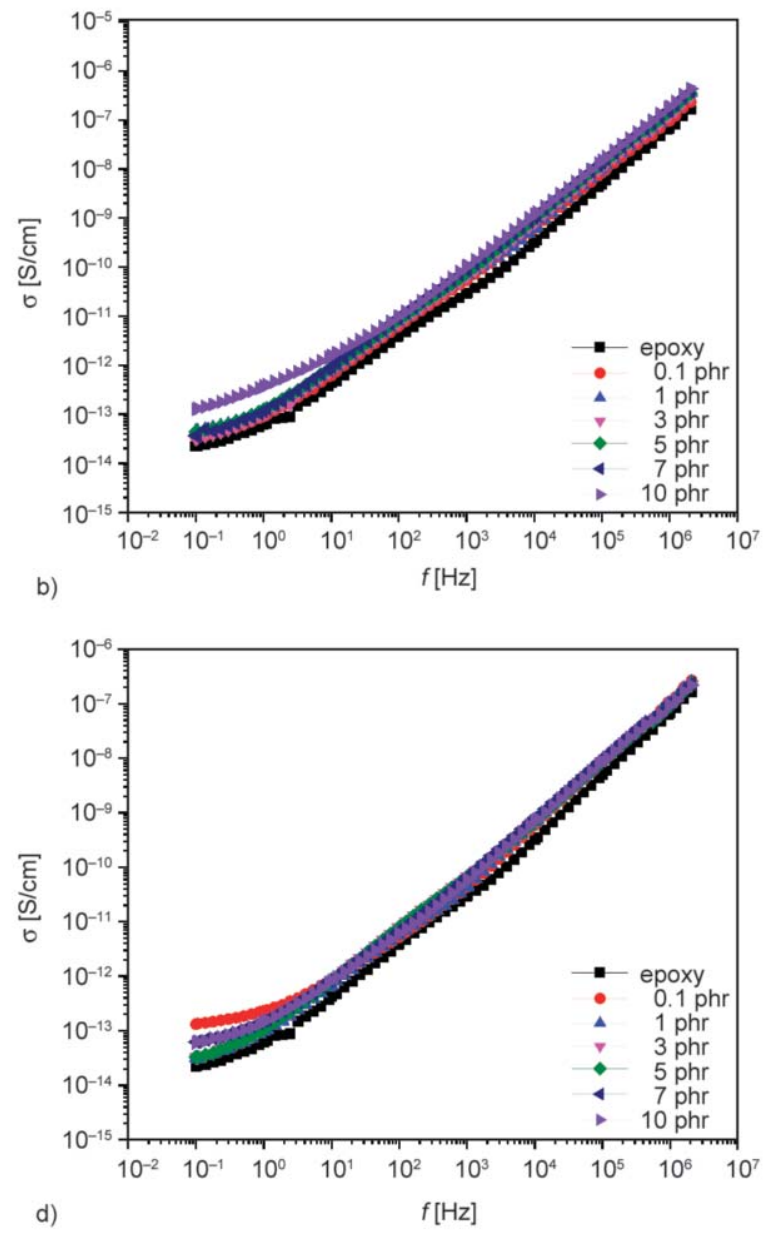

Figure 5. AC conductivity as a function of frequency, at $30^{\circ} \mathrm{C}$, for the nanocomposites with: (a) carbon black, (b) MWCNT, (c) $\mathrm{GnP}$ and (d) nanodiamonds nanoparticles.

is formed within the composite allowing the charge migration [26-28]. The transition from the insulating to the conductive behaviour is mathematically expressed by Equation (4):

$\sigma \approx \sigma_{0}\left(P-P_{\mathrm{C}}\right)^{\mathrm{t}}$

where $\sigma_{0}$ is the pre-exponential factor, $P$ is the content of the conductive phase, $P_{\mathrm{C}}$ is the critical concentration or percolation threshold, and $t$ is an exponent related to the dimensionality of the conduction process. According to classical percolation theory, the increase in filler content brings the conductive inclusions closer, and at the percolation threshold, conductive inclusions form a path via their geometrical contacts.

All the recorded dielectric relaxation mechanisms are evident in the comparative plots of the loss tangent versus frequency at $160^{\circ} \mathrm{C}$ for all systems depicted in Figure 6. From high to low frequencies these mechanisms are assigned to the reorientation of small polar side groups of the polymer chain, the glass to rubber transition and the interfacial polarization.

In nanocomposites with filler contents higher than the critical concentration, IP is not present since the mobile charges don't accumulate at the interface between the filler and the matrix but flow through the formed conductive paths percolating the whole specimen, thus contributing to electrical conduction. Furthermore, for the same systems, the high values of the loss tangent 'mask' $\beta$-relaxation process, while the excess number of nanoparticles hinders the reorientation of small polar side groups of the polymer chain.

Insets depict the imaginary part of electric modulus versus frequency for all systems at $160^{\circ} \mathrm{C}$. The peak formed at the spectra of all systems is associated with the $\alpha$-relaxation process of the polymer matrix. The shift of the peak to higher or lower frequencies indicates the interactions that occur inside the material. For the nanocomposites filled with MWCNT and carbon black, loss peaks shift to higher frequencies with filler content. This is an indication of 

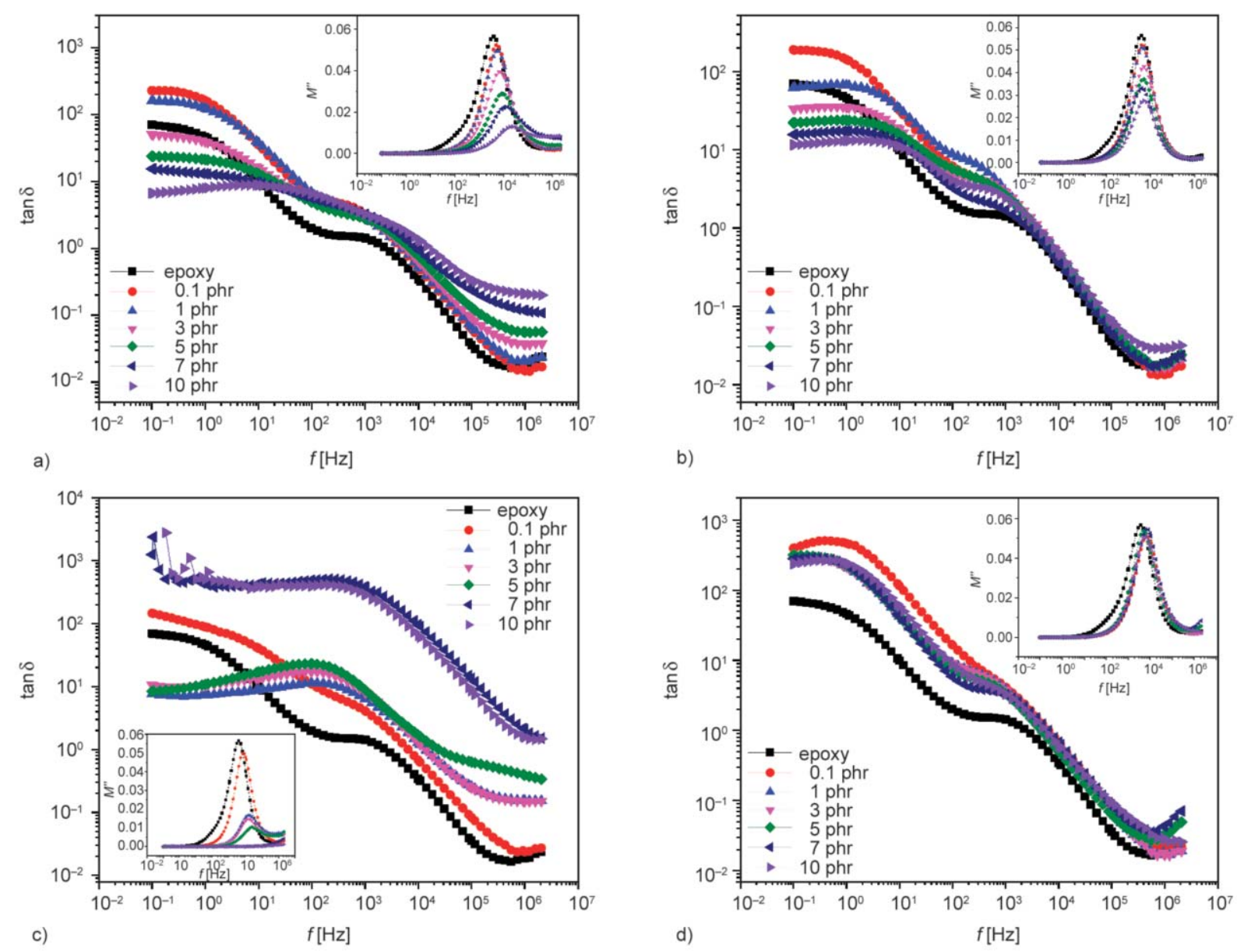

Figure 6. Loss tangent as a function of frequency, at $160^{\circ} \mathrm{C}$, for the nanocomposites with: (a) carbon black, (b) MWCNT, (c) $\mathrm{GnP}$ and (d) nanodiamonds nanoparticles. Insets depict the imaginary part of the electric modulus as a function of frequency, at $160^{\circ} \mathrm{C}$ for the respective nanocomposites.

strong inter-particle interactions that enhance chain flexibility facilitating the relaxation process. In the case of nanocomposites with nanodiamonds, $\alpha$-relaxation shifts to higher frequencies with respect to the pure matrix but remains unaffected by any further increase in filler loading. This is an indication that the strength of the particle-particle interactions doesn't grow with the increase of filler nanoparticles content. The loss peaks for the GnP filled nanocomposites remain invariable with filler concentration. This behaviour is attributed to the good adhesion of the filler with the polymer matrix and an equilibrium between the macromolecules-particles and particlesparticles interactions [29, 30].

The variations of the electrical response induced by the different carbon allotropes are clearly illustrated at Figure 7, which depicts the real part of dielectric permittivity (as an inset) and the $\mathrm{AC}$ conductivity versus frequency respectively, at $30^{\circ} \mathrm{C}$ for nanocomposites with different nanoinclusions at the same filler content. $\varepsilon^{\prime}$ and $\sigma_{\mathrm{AC}}$ increase with filler loading for all examined systems. The MWCNT nanocomposites attain significantly higher values of $\varepsilon^{\prime}$ and $\sigma_{\mathrm{AC}}$ even at low filler content. All other systems attain moderate values with no considerable differences between their electrical responses. At intermediate filler concentrations, Figure 7a, MWCNT systems continue to attain the highest values of both conductivity and permittivity. At the same concentration for the carbon black nanocomposites, there is a significant enhancement in the electrical properties signifying the approach of the critical concentration. At high filler content, Figure 7b, the MWCNT systems have already transitioned to the conductive behaviour, while the high values of the $\varepsilon^{\prime}$ and $\sigma_{\mathrm{AC}}$ attained by the carbon black filled systems indicate that their percolation threshold has been exceeded. The higher electrical conductivity (and therefore also permittivity) exhibited by MWCNT nanocomposites is mainly attributed to the high aspect ratio and rod-like morphology of MWCNTs, which favors the formation of a highly interconnected filler network within 

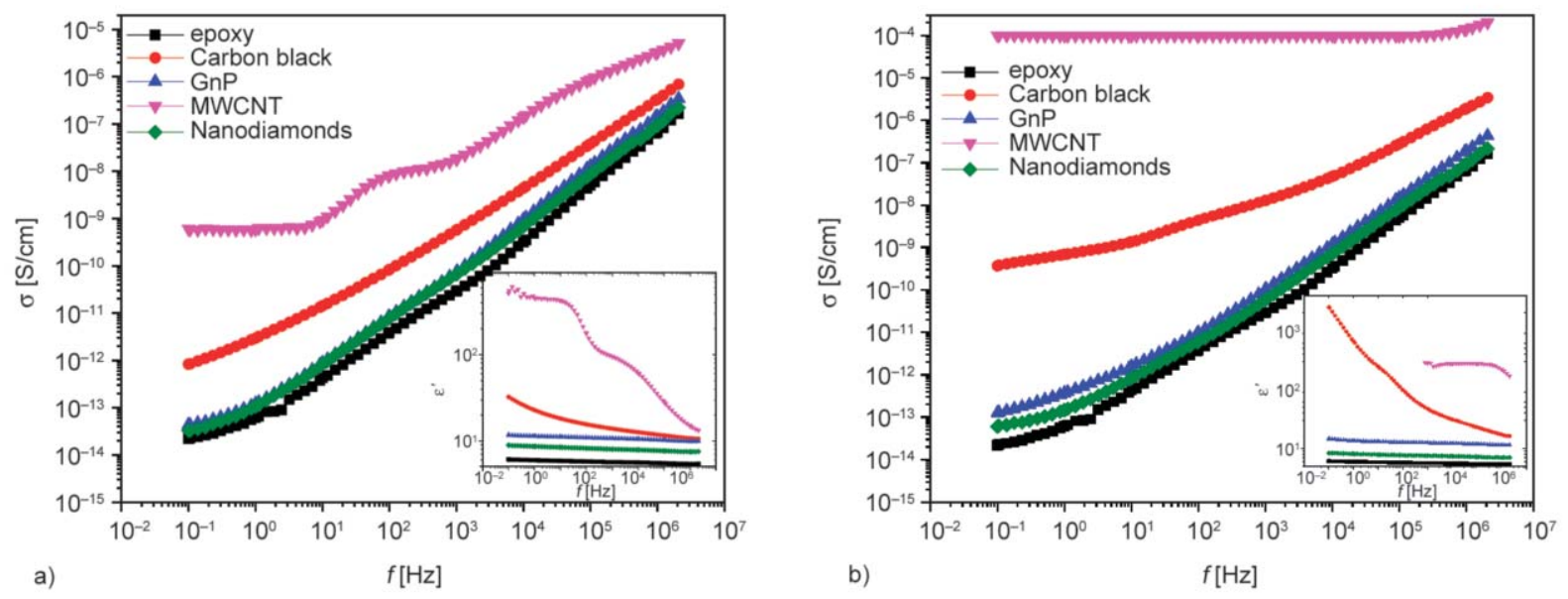

Figure 7. AC conductivity as a function of frequency, at $30^{\circ} \mathrm{C}$, for all examined nanocomposites with: (a) $5 \mathrm{phr}$, and (b) $10 \mathrm{phr}$ filler content. Insets depict the real part of permittivity at $30^{\circ} \mathrm{C}$ for the same systems.

the polymer matrix, as also indicated in Figure 1c. The other two systems remain at the insulating behaviour. GnP filled nanocomposites achieve higher values of both permittivity and conductivity than the nanodiamond composites. Pristine graphene is a defect-free monolayer of carbon atoms in $\mathrm{sp}^{2}$ hybridization with high electrical conductivity. However, the presence of defects in planar graphene stacks lowers their conductivity significantly due to the destruction of the $\mathrm{sp}^{2}$ structure [3,18]. Additional factors reducing conductivity could be the interactions with the molecular environment and surface charge trapping [3, 18]. LRS spectra of the employed GnPs provided evidence for the presence of $\mathrm{sp}^{3} \mathrm{de}-$ fects, via the recorded peak of $\mathrm{D}$ band at approximately $1350 \mathrm{~cm}^{-1}$. The latter could be considered as responsible for the achieved low conductivity values in the $\mathrm{GnP} /$ epoxy systems.

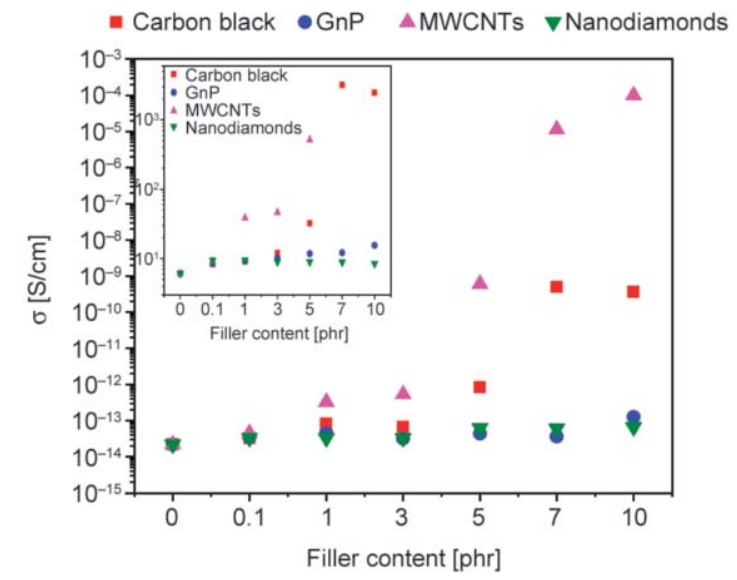

Figure 8. AC conductivity and real part of permittivity (inset) versus filler content at $30^{\circ} \mathrm{C}$ and $0.1 \mathrm{~Hz}$, for all examined systems.
The values of the real part of dielectric permittivity (inset) and $\mathrm{AC}$ conductivity as a function of filler content at $30^{\circ} \mathrm{C}$ and $0.1 \mathrm{~Hz}$ for all examined systems are depicted in Figure 8. MWCNT nanocomposites exhibit the highest values of both $\varepsilon^{\prime}$ and $\sigma_{\mathrm{AC}}$ for all examined filler concentrations followed by the carbon black and GnP filled nanocomposites. Nanodiamond filled systems showed poor electrical response for all examined concentrations. The high values of conductivity of MWCNT and carbon black systems (more than nine and four orders of magnitude, respectively, compared to the epoxy matrix) along with the rapid increase of $\varepsilon^{\prime}$ and $\sigma_{\mathrm{AC}}$ within a marginal increase of filler loading, is a strong indication of exceeding the critical threshold and transitioning from the insulating to the conducting state.

According to the classical percolation theory, conductive inclusions are considered as not interacting hard spheres, randomly dispersed within the insulating matrix, and the critical concentration and exponent depend only on the geometrical characteristics of the inclusions and the type of the conduction process $(1 \mathrm{D}, 2 \mathrm{D}, 3 \mathrm{D})$, respectively. The abrupt rise of conductivity appears as the result of the transition from the insulating state, where limited contacts between conductive sites exist, to the state where a conductive network is formed, via physical contacts of conductive inclusions [26-28, 31, 32].

Critical concentration and exponent can be determined by fitting experimental data via Equation (4) in its logarithmic form as expressed by Equation (5):

$\log \sigma \approx t\left[\log \left(P-P_{\mathrm{C}}\right)\right]$ 
Determined values of critical concentration $P_{\mathrm{C}}$ and exponent $t$ were found to be $4.71 \% \mathrm{w} / \mathrm{w}$ (equivalent to $4.95 \mathrm{phr}$ ) and 2.61 respectively for the MWCNT systems and $5.26 \% \mathrm{w} / \mathrm{w}(5.56 \mathrm{phr})$ and 1.41 for the carbon black systems.

Charge transport properties in polymer nanocomposites depend on several parameters like the shape of the inclusions, the interparticle interactions, the macromolecules-particles interactions, the dispersion of the nanofiller, the polymer matrix and the fabrication method. Figure 9a depicts the variation of conductivity versus the reciprocal temperature for the MWCNT nanocomposites. Fitting experimental data to an Arrhenius type equation allows the determination of the activation energy for thermally activated conduction mechanisms occurring in dielectric materials $[11,33]$. This behaviour is mathematically expressed by Equation (6):

$$
\sigma(T)=\sigma^{0} e^{-\frac{E_{A}}{k_{B} T}}
$$

where $\sigma^{0}$ is a pre-exponential factor, $E_{\mathrm{A}}$ is the activation energy and $k_{\mathrm{B}}$ the Boltzmann constant. The same fitting procedure was applied to all studied systems. The determined values of activation energy are listed in Table 1, indicating the classification of the examined systems into two different sets regarding their conductive response. The nanocomposites reinforced with $\mathrm{GnP}$ and nanodiamonds do not exhibit a significant variation in their activation energy values with filler content. The latter, as well as the type of frequency dependence of conductivity (see Figure $5 \mathrm{~b}$ and $5 \mathrm{~d}$ ), provide indications that hopping conductivity is the predominant conduction mechanism in these nanocomposites [28, 31, 32, 34], which seems to be independent of the reinforcing phase concentration.

On the other hand, nanocomposites filled with MWCNT and carbon black nanoparticles, attain values of activation energy which decrease drastically with filler content. For low filler content, the nanocomposites exhibit insulating behaviour with high activation energies (exceeding $1 \mathrm{eV}$ ), characteristic for the insulating polymer matrix. Notably, that at low filler content activation energy values, for all examined systems, are very close. Conductivity increases gradually with filler content since limited conductive paths are formed and the distance between inclusions decreases, diminishing, as a result, the corresponding values of activation energy. With

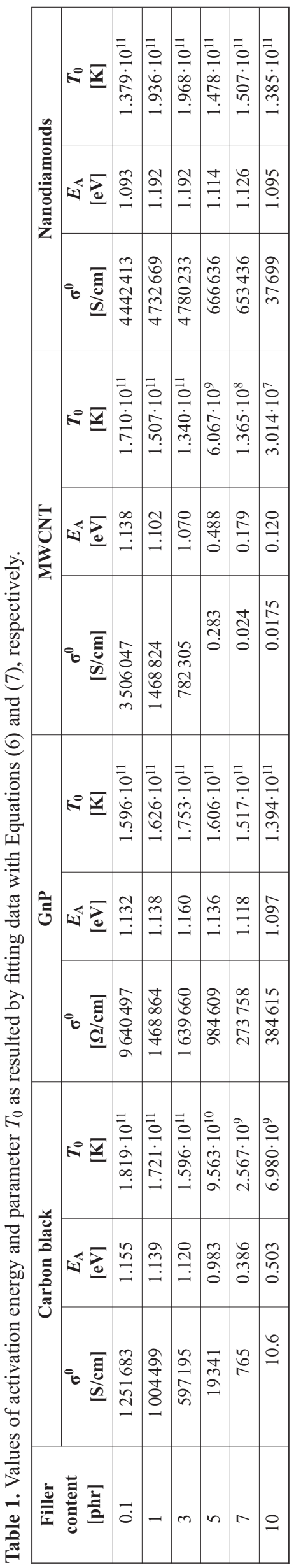


further increase of the filler content, an abrupt increase of conductivity occurs accompanied by a rapid decrease in the values of activation energy, which approach zero, signifying the insulator-to-conductor transition. The transition from the insulating to the conductive behaviour, in tandem with the significant variation of activation energy, could imply an alternation in the charge transport process or the introduction of an additional one [28, 31, 32, 34]. Inset in Figure 9a describes visually the variation of activation energy for all studied systems. Values of the pre-exponential factor $\sigma^{0}$, also listed in Table 1 , diminish with the increase of carbon content. The decrease of $\sigma^{0}$ becomes dramatic in the case of the percolative systems indicating indirectly the existence of an additional charge transport mechanism, most probably via physical contacts $[32,34]$.

According to classical percolation theory, above the critical concentration, nanoinclusions form a conductive path by geometrical contacts, and charges are free to migrate through the nanocomposite. This type of conduction approaches the metallic conduction mechanism and Equation (6) should not be applicable to experimental data. However, Figure 9a and values in Table 1 denote that the validity of Equation (6) upon data holds even at high filler loading. By these means, it can be suggested that hopping conductivity is the main charge transport mechanism of the examined systems, below the critical concentration. Above the percolation threshold, another conduction mechanism is introduced, resembling the metallic type conduction, due to the charge migration via physical contacts of the conductive sites.

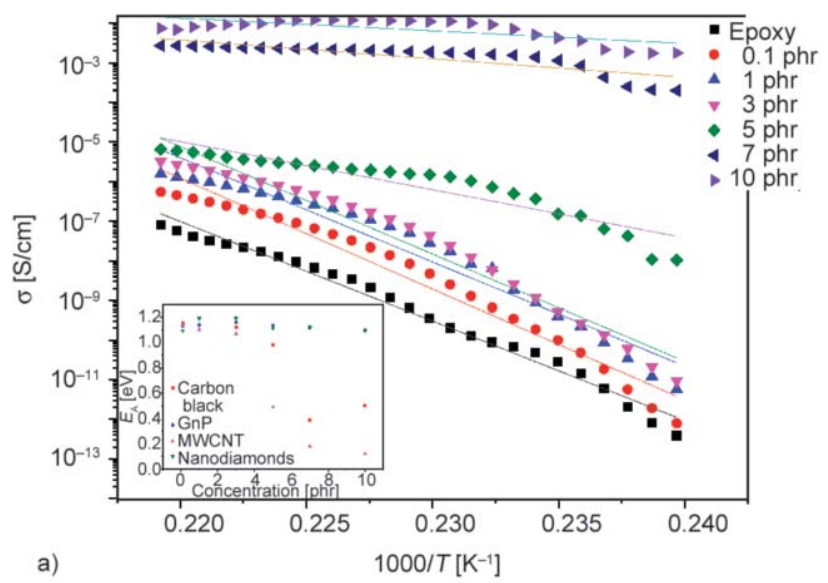

This conduction mechanism is considered responsible for the reduction of activation energy. However, as explained previously, this mechanism does not prevail in the overall conduction process and co-exists with hopping conductivity. The term hopping includes both jumps over a potential barrier and quantum mechanical tunneling [35]. Aiming to verify the occurrence of hopping conductivity in the examined systems, the applicability of Variable Range Hopping Model (VRH), originally proposed by Mott [36, 37], on our experimental data was examined. According to the VRH mechanism charge carriers hop from a localized state to a nearby localized state of different energy, or to a localized state of similar energy with spatial separation from the initial site. The dependence of conductivity on temperature is expressed mathematically by Equation (7):

$\sigma(T)=\sigma_{0} \mathrm{e}^{-\left(\frac{\mathrm{T}_{0}}{\mathrm{~T}}\right)^{\gamma}}$

where the parameter $\sigma_{0}$ can be considered as the limiting value of conductivity at infinite temperature, $T_{0}$ is the characteristic temperature that determines the thermally activated hopping among localized states at different energies and considered as a measure of disorder, and the exponent $\gamma$ is related to the dimensionality $d$ of the transport process via $\gamma=1 /(d+1)$, where $d=1,2,3$.

In Figure 9b experimental data have been plotted in the form of $\log \left(\sigma T^{1 / 2}\right)$ versus $\left(T^{-\gamma}\right)$, which is used for examining the applicability of VRH model [39-41], for $\gamma=1 / 4$ since the samples are bulk polymer specimens incorporating randomly distributed conductive

Figure 9. (a) AC conductivity as a function of the reciprocal temperature for the nanocomposites with MWCNT filler content at $0.1 \mathrm{~Hz}$. (b) AC conductivity of all examined nanocomposites with $10 \mathrm{phr}$ filler content at $0.1 \mathrm{~Hz}$, as described by VRH model. Insets depict the values of the (a) activation energy and (b) $T_{0}$ parameter as derived from the linear fits for the Arrhenius equation and VRH model respectively. 
particles characterized by a 3D conduction process. The well-fitted curves confirm hopping as the charge transport mechanism. The values of the parameter $T_{0}$, derived from the linear fits of Figure $9 \mathrm{a}$, are listed in Table 1.

Inset of Figure $9 \mathrm{~b}$ depicts the variation of parameter $T_{0}$ as a function of filler content for all examined systems. The results for the parameter $T_{0}$ showcase the same behaviour with the results for the activation energy for all studied systems. In both cases, there is slight differentiation in the values for the GnP and nanodiamond composites, while a transition from high values, reflecting limited conductivity, to lower (4 orders of magnitude for the MWCNT systems) ones with increasing filler content appears in the same range in both parameters for the MWCNT and carbon black filled systems. This is a clear indication that for the composites with $\mathrm{GnP}$ and nanodiamonds, along with the low filler content range for the other two systems, hopping is the principal charge transport mechanism. By increasing the carbon black and MWCNT content, conductive sites are spreading and approaching, thus enhancing the metallic type contribution, while at the same time the short distance between of them facilitates the hopping mechanism. This cooperative effect is responsible for the abrupt increase in the conductivity values of the nanocomposites. Above the percolation threshold, the deviation of the fitting quality factor $\left(R^{2}\right)$ from unity in Figure $9 \mathrm{~b}$ signifies the reduced contribution of the hopping transport mechanism. Although, the positive values of activation energy denote that conduction does not take place exclusively via a conductive path through physical contacts between nanoinclusions $[31,32]$.

\section{Conclusions}

In the present study, different sets of polymer nanocomposites employing a variety of carbon allotropes as filler were developed and their electrical properties were investigated by means of dielectric spectroscopy. The addition of filler was found to enhance the values of the real part of dielectric permittivity and ac conductivity in all cases, except the nanodiamond filled systems where both values increased for low concentrations but remained stable or even decreased with further filler loading. Three distinct relaxation mechanisms were recorded in the spectra of all systems originating from the fillers and the polymer matrix and were attributed to interfacial polarization, glass to rubber transition and reorientation of polar side groups of the polymer matrix. In the carbon black and MWCNT systems an abrupt increase in the permittivity and conductivity values was recorded above a critical filler concentration signifying the transition from the insulating to the conducting state. The conduction processes in all four sets of nanocomposites have been investigated in the present study. Conductivity data were analyzed with regard to the percolation theory, and the percolation threshold was determined. The dependence of conductivity on temperature and the Variable Range Hopping model were employed in order to reveal the charge transport mechanisms below, in the vicinity, and above the critical concentration. Below the percolation threshold and in the limit of the transition zone, hopping conductivity appears to be the principal charge transport mechanism. At concentrations higher than the threshold there is coexistence between hopping and metallic type conduction.

\section{Acknowledgements}

The present research work was supported by the Hellenic Foundation for Research and Innovation (HFRI) and the General Secretariat for Research and Technology (GSRT) under the HFRI PhD Fellowship grant (GA. no.2327).

Authors wish to acknowledge the Laboratory of Applied Molecular Spectroscopy (LAMS) of FORTH/ICE-HT; more specifically Dr. G.A. Voyiatzis (Research Director) for providing access to the Raman spectroscopy experimental facilities and Dr. K.S. Andrikopoulos for his support regarding the related experiments.

\section{References}

[1] Thostenson E. T., Li C., Chou T-W.: Nanocomposites in context. Composites Science and Technology, 65, 491-516 (2005). https://doi.org/10.1016/j.compscitech.2004.11.003

[2] Moniruzzaman M., Winey K. I.: Polymer nanocomposites containing carbon nanotubes. Macromolecules, 39, 5194-5205 (2006). https://doi.org/10.1021/ma060733p

[3] Singh V., Joung D., Zhai L., Das S., Khondaker S. I., Seal S.: Graphene based materials: Past, present and future. Progress in Materials Science, 56, 1178-1271 (2011).

https://doi.org/10.1016/j.pmatsci.2011.03.003

[4] Roy N., Sengupta R., Bhowmick A. K.: Modifications of carbon for polymer composites and nanocomposites. Progress in Polymer Science, 37, 781-819 (2012). https://doi.org/10.1016/j.progpolymsci.2012.02.002 
[5] Tantis I., Psarras G. C., Tasis D.: Functionalized graphene - poly(vinyl alcohol) nanocomposites: Physical and dielectric properties. Express Polymer Letters, 6, 283-292 (2012). https://doi.org/10.3144/expresspolymlett.2012.31

[6] Xu J-Z., Zhong G-J., Hsiao B. S., Fu Q., Li Z-M.: Lowdimensional carbonaceous nanofiller induced polymer crystallization. Progress in Polymer Science, 39, 555593 (2014).

https://doi.org/10.1016/j.progpolymsci.2013.06.005

[7] Psarras G. C.: Nanographite-polymer composites. in 'Carbon nanomaterials sourcebook: Nanoparticles, nanocapsules, nanofibers, nanoporous structures, and nanocomposites' (ed.: Sattler K. D.) CRC Press, Boca Raton, Vol 2, 647-673 (2016).

https://doi.org/10.1201/9781315371337

[8] Toldy A.: Flame retardancy of carbon fibre reinforced composites. Express Polymer Letters, 12, 186 (2018). https://doi.org/10.3144/expresspolymlett.2018.17

[9] Wu T. H., Foyet A., Kodentsov A., van der Ven L. G. J., van Benthem R. A. T. M., de With G.: Curing and percolation for carbon black-epoxy-amine nanocomposites. Composites Science and Technology, 181, 107672/1107672/8 (2019).

https://doi.org/10.1016/j.compscitech.2019.05.029

[10] Georgakilas V., Perman J. A., Tucek J., Zboril R.: Broad family of carbon nanoallotropes: Classification, chemistry, and applications of fullerenes, carbon dots, nanotubes, graphene, nanodiamonds, and combined superstructures. Chemical Reviews, 115, 4744-4822 (2015). https://doi.org/10.1021/cr500304f

[11] Psarras G. C.: Conductivity and dielectric characterization of polymer nanocomposites. in 'Physical properties and applications of polymer nanocomposites' (eds.: Tjong S. C., Mai Y-W.) Woodhead, Cambridge, 31-69 (2010).

https://doi.org/10.1533/9780857090249.1.31

[12] Sengupta R., Bhattacharya M., Bandyopadhyay S., Bhowmick A. K.: A review on the mechanical and electrical properties of graphite and modified graphite reinforced polymer composites. Progress in Polymer Science, 36, 638-670 (2011).

https://doi.org/10.1016/j.progpolymsci.2010.11.003

[13] Rahman A., Ali I., Al Zahrani S. M., Eleithy R. H.: A review of the applications of nanocarbon polymer composites. Nano, 06, 185-203 (2011).

https://doi.org/10.1142/S179329201100255X

[14] Agnelli S., Pandini S., Torricelli F., Romele P., Serafini A., Barbera V., Galimberti M.: Anisotropic properties of elastomeric nanocomposites based on natural rubber and $\mathrm{sp}^{2}$ carbon allotropes. Express Polymer Letters, 12, 713-730 (2018).

https://doi.org/10.3144/expresspolymlett.2018.61

[15] Gumede T. P., Luyt A. S., Müller A. J.: Review on PCL, PBS, and PCL/PBS blends containing carbon nanotubes. Express Polymer Letters, 12, 505-529 (2018). https://doi.org/10.3144/expresspolymlett.2018.43
[16] Vavouliotis A., Fiamegou E., Karapappas P., Psarras G. C., Kostopoulos V.: DC and AC conductivity in epoxy resin/multiwall carbon nanotubes percolative system. Polymer Composites, 31, 1874-1880 (2010). https://doi.org/10.1002/pc.20981

[17] Saravanan N., Rajasekar R., Mahalakshmi S., Sathishkumar T., Sasikumar K., Sahoo S.: Graphene and modified graphene-based polymer nanocomposites - A review. Journal of Reinforced Plastics and Composites, 33, 1158-1170 (2014).

https://doi.org/10.1177/0731684414524847

[18] Galpaya D., Wang M., Liu M., Motta N., Waclawik E., Yan C.: Recent advances in fabrication and characterization of graphene-polymer nanocomposites. Graphene, 01, 30-49 (2012). https://doi.org/10.4236/graphene.2012.12005

[19] Kingston C., Zepp R., Andrady A., Boverhof D., Fehir R., Hawkins D., Roberts J., Sayre P., Shelton B., Sultan Y., Vejins V., Wohlleben W.: Release characteristics of selected carbon nanotube polymer composites. Carbon, 68, 33-57 (2014). https://doi.org/10.1016/j.carbon.2013.11.042

[20] Ferrari A. C., Meyer J. C., Scardaci V., Casiraghi C., Lazzeri M., Mauri F., Piscanec S., Jiang D., Novoselov K. S., Roth S., Geim A. K.: Raman spectrum of graphene and graphene layers. Physical Review Letters, 97, 187401/1-187401/4 (2006).

https://doi.org/10.1103/PhysRevLett.97.187401

[21] Tsangaris G. M., Psarras G. C., Kouloumbi N.: Electric modulus and interfacial polarization in composite polymeric systems. Journal of Materials Science, 33, 2027 2037 (1998). https://doi.org/10.1023/A:1004398514901

[22] Gatos K. G., Martínez Alcázar J. G., Psarras G. C., Thomann R., Karger-Kocsis J.: Polyurethane latex/water dispersible boehmite alumina nanocomposites: Thermal, mechanical and dielectrical properties. Composites Science and Technology, 67, 157-167 (2007). https://doi.org/10.1016/j.compscitech.2006.07.025

[23] Psarras G. C., Gatos K. G., Karahaliou P. K., Georga S. N., Krontiras C. A., Karger-Kocsis J.: Relaxation phenomena in rubber/layered silicate nanocomposites. Express Polymer Letters, 1, 837-845 (2007). https://doi.org/10.3144/expresspolymlett.2007.116

[24] Hernández M., Carretero-González J., Verdejo R., Ezquerra T. A., López-Manchado M. A.: Molecular dynamics of natural rubber/layered silicate nanocomposites as studied by dielectric relaxation spectroscopy. Macromolecules, 43, 643-651 (2010).

https://doi.org/10.1021/ma902379t

[25] Psarras G. C., Siengchin S., Karahaliou P. K., Georga S. N., Krontiras C. A., Karger-Kocsis J.: Dielectric relaxation phenomena and dynamics in polyoxymethylene/polyurethane/alumina hybrid nanocomposites. Polymer International, 60, 1715-1721 (2011).

https://doi.org/10.1002/pi.3136 
[26] Lux F.: Models proposed to explain the electrical conductivity of mixtures made of conductive and insulating materials. Journal of Materials Science, 28, 285-301 (1993). https://doi.org/10.1007/BF00357799

[27] Zallen R.: The physics of amorphous solids. Wiley, New York (2004).

[28] Psarras G. C.: Hopping conductivity in polymer matrix-metal particles composites. Composites Part A: Applied Science and Manufacturing, 37, 1545-1553 (2006). https://doi.org/10.1016/j.compositesa.2005.11.004

[29] Vryonis O., Anastassopoulos D. L., Vradis A. A., Psarras G. C.: Dielectric response and molecular dynamics in epoxy-BaSrTiO${ }_{3}$ nanocomposites: Effect of nanofiller loading. Polymer, 95, 82-90 (2016).

https://doi.org/10.1016/j.polymer.2016.04.050

[30] Sanida A., Stavropoulos S. G., Speliotis T., Psarras G. C.: Development, characterization, energy storage and interface dielectric properties in $\mathrm{SrFe}_{12} \mathrm{O}_{19}$ /epoxy nanocomposites. Polymer, 120, 73-81 (2017). https://doi.org/10.1016/j.polymer.2017.05.043

[31] Psarras G. C.: Charge transport properties in carbon black/polymer composites. Journal of Polymer Science Part B: Polymer Physics, 45, 2535-2545 (2007). https://doi.org/10.1002/polb.21278

[32] Psarras G. C.: Conduction processes in percolative epoxy resin/silver particles composites. Science of Advanced Materials, 1, 101-106 (2009). https://doi.org/10.1166/sam.2009.1015

[33] Kremer F., Schönhals A.: Broadband dielectric spectroscopy. Springer, Berlin (2003).
[34] Pontikopoulos P. L., Psarras G. C.: Dynamic percolation and dielectric response in multiwall carbon nanotubes/poly(ethylene oxide) composites. Science of Advanced Materials, 5, 14-20 (2013). https://doi.org/10.1166/sam.2013.1425

[35] Böttger H., Bryksin V. V.: Hopping conduction in solids. Akad-Verlag, Berlin (1985).

[36] Mott N. F.: Conduction in non-crystalline materials. Philosophical Magazine, 19, 835-852 (1969). https://doi.org/10.1080/14786436908216338

[37] Mott N. F.: Metal-insulator transitions. Taylor and Francis, London (1990).

[38] Mott N. F., Davis E. A.: Electronic processes in noncrystalline materials. Oxford University Press, New York, (2012).

[39] Zuo F., Angelopoulos M., Macdiarmid A. G., Epstein A. J.: AC conductivity of emeraldine polymer. Physical Review B, 39, 3570-3578 (1989). https://doi.org/10.1103/PhysRevB.39.3570

[40] Aguilar-Hernández J., Potje-Kamloth K.: Evaluation of the electrical conductivity of polypyrrole polymer composites. Journal of Physics D: Applied Physics, 34, 1700-1711 (2001). https://doi.org/10.1088/0022-3727/34/11/323

[41] Epstein A. J., Rommelmann H., Bigelow R., Gibson H. W., Hoffmann D. M., Tanner D. B.: Role of solitons in nearly metallic polyacetylene. Physical Review Letters, 50, 1866-1869 (1983). https://doi.org/10.1103/PhysRevLett.50.1866 